\title{
Phase noise reduction by self-phase locking in semiconductor lasers using phase conjugate feedback
}

Petersen, Lykke; Gliese, Ulrik Bo; Nielsen, Torben Nørskov

Published in:

I E E E Journal of Quantum Electronics

Link to article, DOI:

$10.1109 / 3.333704$

Publication date:

1994

Document Version

Publisher's PDF, also known as Version of record

Link back to DTU Orbit

Citation (APA):

Petersen, L., Gliese, U. B., \& Nielsen, T. N. (1994). Phase noise reduction by self-phase locking in semiconductor lasers using phase conjugate feedback. I E E E Journal of Quantum Electronics, 30(11), 25262533. https://doi.org/10.1109/3.333704

\section{General rights}

Copyright and moral rights for the publications made accessible in the public portal are retained by the authors and/or other copyright owners and it is a condition of accessing publications that users recognise and abide by the legal requirements associated with these rights.

- Users may download and print one copy of any publication from the public portal for the purpose of private study or research.

- You may not further distribute the material or use it for any profit-making activity or commercial gain

- You may freely distribute the URL identifying the publication in the public portal 


\title{
Phase Noise Reduction by Self-Phase Locking in Semiconductor Lasers Using Phase Conjugate Feedback
}

\author{
Lykke Petersen, Ulrik Gliese, and Torben Nørskov Nielsen
}

\begin{abstract}
A theoretical analysis of the behavior of the frequency/phase noise of semiconductor lasers with external phase conjugate feedback is presented. It is shown that the frequency noise is drastically reduced even for lasers with butt-coupled phase conjugate mirrors. In this laser system, the phase noise takes a finite-low value corresponding to a state of first-order self-phase locking of the laser. As a result, the spectral shape of the laser signal does not remain Lorentzian but collapses around the carrier to a delta function with a close to carrier noise level of less than $-137 \mathrm{dBc} / \mathrm{Hz}$. The total phase variance of this laser signal, in a $20 \mathrm{GHz}$ noise bandwidth, is less than $0.002 \mathrm{rad}^{2}$.
\end{abstract}

\section{INTRODUCTION}

$\mathbf{M}$ ANY coherent optical systems such as optical communication systems, optical microwave subcarrier systems, optical signal-processing systems, and optical measurement systems require lasers with very low phase noise. At the same time, the use of semiconductor lasers are desirable in many of these applications since they are compact and have potential for monolithic optoelectronic integration. Semiconductor lasers, however, have a significant amount of phase noise, i.e., linewidths in the order of $10 \mathrm{kHz}$ to $100 \mathrm{MHz}$. Methods for efficient reduction of this phase noise are therefore very important.

Direct phase noise reduction has been obtained by conventional external optical feedback from long external cavities [1], by locking to atomic lines [2], and by locking to interferometers [3]. Indirect phase noise reduction has been obtained in dual laser signal applications by noise correlation using frequency shifting [4], by optical injection locking [5], and by optical phase locking [6]. Here, we investigate the use of optical phase conjugate feedback (PCF) to obtain direct phase noise reduction in semiconductor lasers.

The use of external PCF in semiconductor lasers have gained an increasing interest. This is because the reflected beam from the external phase conjugate mirror (PCM), due to phase reversal, traces exactly back along the incoming beam as shown in Fig. 1. The consequence of this is a mechanically much more stable system which within certain limits is self-aligning [7]. In this respect, the PCM has, until now, been used for implementation of mechanically stable

Manuscript received March 22, 1994.

The authors are with the Center for Broadband Telecommunications, Electromagnetics Institute, Technical University of Denmark, Lyngby, Denmark, DK-2800.

IEEE Log Number 9403497
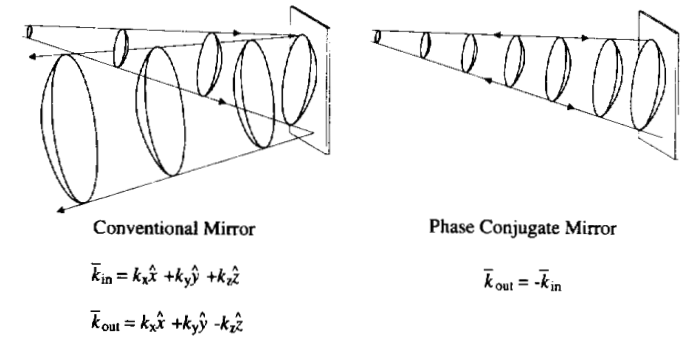

Phase Conjugate Mirror

$\bar{k}_{\text {out }}=-\bar{k}_{\text {in }}$

Fig. 1. Illustration of the phase front reversal property of the phase conjugate mirror where $k$ describes the wave vector.

external cavity semiconductor lasers [8], [9] as well as for efficient and mechanically stable injection locking of laser diode arrays [10]-[12].

The theoretical analysis of semiconductor lasers with PCF is still sparse and many aspects need to be analyzed. Until now, aspects of semiconductor laser far field characteristics [13], dynamics [14]-[17], and noise [16]-[19] have been analyzed in connection with PCF from long (centimeters to meters) external cavities. In this paper, we contribute to the understanding of both DFB and FP semiconductor lasers with external PCF by performing an analysis of the phase noise behavior considering both long and short (submillimetre) external cavity lengths.

Following the basic theory of phase conjugate resonators [7], it should be possible to obtain an improved frequency/phase noise performance of semiconductor lasers using PCF. Here, it is shown that even for butt-coupled PCMs, the PCF drastically reduces the phase noise of the semiconductor laser corresponding to a state of first-order self-phase locking. This results in a significant spectral narrowing yielding a very clean carrier peak with a noise level of less than $-137 \mathrm{dBc} / \mathrm{Hz}$ at carrier offset frequencies well below the laser relaxation oscillation.

The paper is outlined as follows. In Section II, the basis of the theoretical analysis is described. Based on this, the expression for the frequency noise of the laser system is described in Section III while the derivation is given in Appendix A. In Section IV, the results of the calculations on the frequency/phase noise are presented and discussed. Finally, in Section V, conclusions are drawn. Appendix B gives an overview of the semiconductor laser data used for the calculations. 


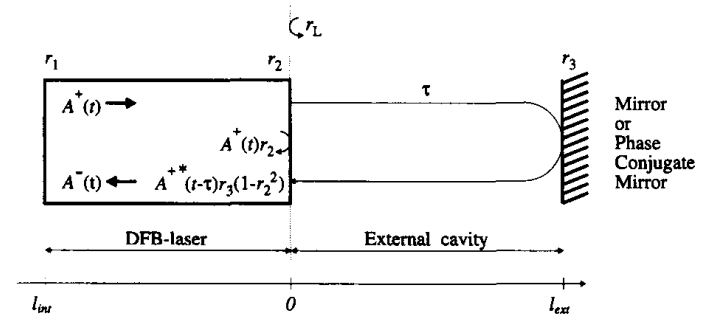

Fig. 2. Schematic of the analyzed semiconductor DFB laser with external feedback from either a conventional mirror or a phase conjugate mirror.

\section{THEORETICAL Description OF THE ANALYZED LASER SYSTEM}

The analyzed laser system consists of a DFB laser with an optional external cavity, as shown in Fig. 2. The length of the DFB laser is given by $l_{\text {int }}$ and the length of the external cavity is given by $l_{\text {ext }}$ which gives a roundtrip time of $\tau$. Further, the left and right facet amplitude reflectivities are given by $r_{1}$ and $r_{2}$, respectively. The feedback from the external cavity is either from a conventional mirror (M) or from a phase conjugate mirror (PCM). In both cases, the feedback fraction is given by $r_{3}$. The theoretical analysis of the system is based on a single-mode transmission-line description which is an extension of [20]. In this paper, only relatively weak feedback fractions $\left(r_{3} \leq 0.3\right)$ from the external cavity are considered, so that multiple reflections in the external cavity can be neglected. Further, the PCM is assumed ideal with instantaneous response generated by degenerate four-wave mixing in a nonlinear medium.

As shown in Fig. 2, the electrical field in the internal laser cavity is divided into a left and right traveling field, and the reference plane at $z=0$ is taken to be just inside the laser facet. Using this formalism, the boundary condition related to the left facet is described by a dynamic field equation:

$$
\begin{aligned}
\frac{d A^{+}(t)}{d t}= & f_{\mathrm{D}}\left[r_{\mathrm{L}} A^{-}(t)-A^{+}(t)\right] \\
& +\frac{1}{2} C G_{N} \Delta N(t) A_{\mathrm{s}}^{+}+F(t)
\end{aligned}
$$

where $\Delta N(t)=N(t)-N_{\mathrm{s}}$ is the deviation of the carrier density from the stationary carrier density $N_{\mathrm{s}}$, and $F(t)$ is a Langevin noise function. The effective amplitude reflection coefficient for the left facet $r_{\mathrm{L}}$ takes both the actual facet reflection $r_{1}$ and the distributed Bragg reflection into account. Further, $r_{\mathrm{L}}$ is independent of the external cavity and is defined as described in [20]. The parameter $f_{\mathrm{D}}$ is given by

$$
f_{\mathrm{D}}=\frac{1}{j \frac{\partial}{\partial \omega} \ln r_{\mathrm{L}}}
$$

and the parameter $C$ is given as

$$
C=\frac{1+j \alpha}{1+j \frac{1}{2} G_{\omega}} .
$$

The differential gain functions are given as

$$
\begin{aligned}
G_{N} & =v_{\mathrm{g}} \frac{\partial g}{\partial N} \\
G_{\omega} & =v_{\mathrm{g}} \frac{\partial g}{\partial \omega}
\end{aligned}
$$

where $v_{\mathrm{g}}$ is the group velocity and $g$ is the modal gain for which we use the model

$$
\begin{aligned}
g(\omega, N)= & \gamma_{N}\left(N-N_{0}\right) \\
& -\gamma_{\omega}\left(\omega-\omega_{\mathrm{p}}\left(N_{0}\right)-\frac{d \omega_{\mathrm{p}}}{d N}\left(N-N_{0}\right)\right)^{2}
\end{aligned}
$$

where $N_{0}$ is the carrier density at transparency, and $\gamma_{N}, \gamma_{\omega}, \omega_{\mathrm{p}}\left(N_{0}\right)$, and $d \omega_{\mathrm{p}} / d N$ are constants. Finally, the linewidth enhancement factor $\alpha$ is given as

$$
\alpha=-2 \cdot \frac{\omega \frac{d n}{d N}}{c_{0} \frac{d g}{d N}} .
$$

Normally, the boundary condition related to the right facet is described by the use of an effective right-facet reflection coefficient [20]. However, this is not possible in the case of external PCF. Instead, the boundary condition is described using two reflection contributions, one for the right facet at time $t$ and one for the external mirror at time $t-\tau$. In this analysis, the boundary condition related to the right facet is divided into two cases. In the case of feedback from a conventional mirror, the boundary condition is given as

$$
A^{-}(t)=r_{2} a^{+}(t)+r_{3}\left(1-r_{2}^{2}\right) A^{+}(t-\tau) e^{-j \omega \tau} .
$$

The boundary condition in the case of feedback from a PCM becomes quite different due to the phase conjugation that takes place in the PCM

$$
A^{-}(t)=r_{2} A^{+}(t)+r_{3}\left(1-r_{2}^{2}\right) A^{+*}(t-\tau) e^{-j \phi_{\mathrm{PCM}}} .
$$

The term $\phi_{\mathrm{PCM}}$ is a constant phase shift occurring at the PCM. Since constant, it has no influence on the phase noise behavior and is therefore, for simplicity, assumed zero.

A complete single-mode description of the laser system in Fig. 2 is obtained by combining (1) as well as (8) or (9) with the rate equation for the carrier density

$$
\frac{d}{d t} N(t)=\frac{I}{e V_{\mathrm{c}}}-R(N)-\frac{v_{\mathrm{g}} g\left(\omega_{s}, N\right) P(t)}{V_{\mathrm{c}}}+F_{N}(t) .
$$

Here, $I$ is the injected current, $V_{\mathrm{c}}$ the volume occupied by the carriers, $R(N)$ is the total rate of spontaneous recombinations per unit volume, $F_{N}(t)$ is the Langevin noise function for the carriers, and $P(t)$ is the number of photons in the laser waveguide in the lasing mode given as

$$
P(t)=\sigma\left(\omega_{\mathrm{s}}, N_{\mathrm{s}}\right)\left|A^{+}(t)\right|^{2}
$$

where

$$
\sigma\left(\omega_{\mathrm{s}}, N_{\mathrm{s}}\right)=\frac{2 \epsilon_{0} n(\omega, N) n_{\mathrm{g}}}{\hbar \omega_{\mathrm{s}}} \int_{-l_{\mathrm{int}}}^{0}\left|\frac{Z_{1}(z)}{Z_{1}^{+}(0)}\right|^{2} d z .
$$


Here, $n_{\mathrm{g}}$ is the group refractive index, $n(\omega, N)$ is the real refractive index and the function $Z_{1}(z)$ is an independent solution to the homogeneous wave equation and is decomposed into a right- and left-traveling component $Z_{1}^{+}$and $Z_{1}^{-}$.

The analysis mainly focuses on DFB semiconductor lasers with weak external feedback. However, the presented model is easily adapted to deal with FP semiconductor lasers simply by setting the coupling coefficient, which is present in $Z_{1}(z)$, to zero. Further, the model can be adapted to treat the case of strong external feedback. This is done by expanding the boundary condition related to the right facet, (8) or (9), to incorporate the desired number of multiple reflections. In this case, however, the derivation of the frequency fluctuation spectrum becomes very complex, and, as shown in Section IV, multiple reflections will not contribute any influence in laser system that turns out to be optimum.

Using the aforementioned transmission line model, the expression for the frequency fluctuation spectrum can be derived as described in Section III.

\section{FReQuenCy Fluctuation SPECTRA}

In order to investigate the phase-noise behavior of semiconductor lasers with PCF, it is necessary to derive an expression describing the frequency fluctuation spectrum and thereby the phase fluctuation spectrum. This is done in Appendix A for semiconductor lasers with external PCF. As will be shown, it turns out that the expression for the frequency fluctuation spectrum of the laser with external PCF is quite similar to that of the laser with external conventional feedback. However, as shown in the following, the behavior is very different.

The frequency fluctuation spectrum of semiconductor lasers is found from the linearized set of Laplace transformed rate equations

$$
\left[\begin{array}{ccc}
s+f_{1} & 2 P_{\mathrm{s}} f_{2} & -C_{\mathrm{r}} G_{N} P_{\mathrm{s}} \\
-\frac{f_{3}}{2 P_{\mathrm{s}}} & s+f_{4} & -\frac{1}{2} C_{i} G_{N} \\
\frac{\omega_{R}^{2}}{G_{N} P_{\mathrm{s}}} & 0 & s+\frac{1}{\tau_{R}}
\end{array}\right]\left[\begin{array}{c}
\delta P \\
\delta \phi \\
\delta N
\end{array}\right]=\left[\begin{array}{c}
F_{P} \\
F_{\phi} \\
F_{N}
\end{array}\right]
$$

where $s=j \omega_{\mathrm{b}}$ and $\omega_{\mathrm{b}}$ is the angular frequency offset from the stationary angular frequency $\omega_{\mathrm{s}}$. Further, $C_{\mathrm{r}}$ and $C_{\mathrm{i}}$ are the real and imaginary parts of $C(3)$, and $\tau_{\mathrm{R}}$ is the relaxation oscillation time constant. The functions $f_{1}-f_{4}$ are characteristic functions of the laser system. From the aforementioned system of equations, the following expression for the double-sideband frequency fluctuation spectrum is derived following the procedure of [21]:

$$
\begin{aligned}
S_{\dot{\phi}}\left(\omega_{\mathrm{b}}\right)= & \frac{R_{\mathrm{sp}} \omega_{\mathrm{b}}^{2}}{2 P_{\mathrm{s}}|D(s)|^{2}}\left\{\left|\left(s+f_{1}\right)\left(s+\frac{1}{\tau_{\mathrm{R}}}\right)+C_{\mathrm{r}} \omega_{\mathrm{R}}^{2}\right|^{2}\right. \\
& \left.+\left|\left(s+\frac{1}{\tau_{\mathrm{R}}}\right) f_{3}-C_{i} \omega_{\mathrm{R}}^{2}\right|^{2}\right\}
\end{aligned}
$$

Here, $R_{\text {sp }}$ is the spontaneous emission rate into the lasing mode and $D(s)$ is the determinant of the matrix in (13).
In (13) and (14), the characteristic functions of the laser system fully describe the influence of the combined laser cavity $^{1}$ on both the intensity and phase noise of the laser system in the frequency domain around steady state $\omega_{\mathrm{s}}$. These functions are derived in Appendix A and constitute the main result of the theoretical description presented here. As seen in (13), the characteristic functions only operate on the photon intensity and phase, and they describe the influence of the combined cavity on

$$
\begin{aligned}
& f_{1}: \text { Intensity noise; } \\
& f_{2}: \text { Phase to intensity converted noise; } \\
& f_{3}: \text { Intensity to phase converted noise; } \\
& f_{4}: \text { Phase noise. }
\end{aligned}
$$

For a semiconductor laser with external feedback from a conventional mirror these functions can be shown to be

$$
\begin{aligned}
f_{1, \mathrm{M}}= & f_{4, \mathrm{M}}=\operatorname{Re}\left(g_{1}\right)-0.5 r_{3}\left(1-r_{2}^{2}\right) \\
& \cdot\left\{g_{2} e^{-j\left(\omega_{\mathrm{s}}+\omega_{\mathrm{b}}\right) \tau}+g_{2}^{*} e^{-j\left(\omega_{\mathrm{s}}-\omega_{\mathrm{b}}\right) \tau}\right\} \\
f_{2, \mathrm{M}}= & f_{3, \mathrm{M}}=-\operatorname{Im}\left(g_{1}\right)+0.5 j r_{3}\left(1-r_{2}^{2}\right) \\
& \cdot\left\{-g_{2} e^{-j\left(\omega_{\mathrm{s}}+\omega_{\mathrm{b}}\right) \tau}+g_{2}^{*} e^{-j\left(\omega_{\mathrm{s}}-\omega_{\mathrm{b}}\right) \tau}\right\}
\end{aligned}
$$

while the characteristic functions for an external cavity with a PCM are

$$
\begin{aligned}
& f_{1, \mathrm{PCM}}=\operatorname{Re}\left(g_{1}\right)-0.5 r_{3}\left(1-r_{2}^{2}\right)\left\{g_{2}+g_{2}^{*}\right\} e^{-j \omega_{\mathrm{b}} \tau} \\
& f_{2, \mathrm{PCM}}=-\operatorname{Im}\left(g_{1}\right)+0.5 j r_{3}\left(1-r_{2}^{2}\right)\left\{g_{2}-g_{2}^{*}\right\} e^{-j \omega_{\mathrm{b}} \tau} \\
& f_{3, \mathrm{PCM}}=-\operatorname{Im}\left(g_{1}\right)-0.5 j r_{3}\left(1-r_{2}^{2}\right)\left\{g_{2}-g_{2}^{*}\right\} e^{-j \omega_{\mathrm{b}} \tau}(19) \\
& f_{4, \mathrm{PCM}}=\operatorname{Re}\left(g_{1}\right)+0.5 r_{3}\left(1-r_{2}^{2}\right)\left\{g_{2}+g_{2}^{*}\right\} e^{-j \omega_{\mathrm{b}} \tau} .
\end{aligned}
$$

In all of these characteristic functions, $g_{1}$ and $g_{2}$ are given as

$$
\begin{aligned}
& g_{1}=f_{\mathrm{D}}\left(1-r_{\mathrm{L}} r_{2}\right) \\
& g_{2}=f_{\mathrm{D}} r_{\mathrm{L}} .
\end{aligned}
$$

As seen, the characteristic functions of the two laser-systems have the same structure, and in both cases, the behavior of a semiconductor laser without external feedback is obtained by setting $r_{3}$ to zero.

A major dissimilarity in the characteristic functions of the two systems is the absence of the steady-state angular frequency $\omega_{\mathrm{s}}$ in the case of PCF. This result is due to the nature of the PCM which ensures that the steady oscillation condition is always fulfilled.

In the case of feedback from a conventional mirror, the characteristic functions are two-by-two alike, $c f$. (15) and (16). This means that the cavity has equal influence on intensity noise and phase noise. However, this is not the case for the system with PCF. The PCM, if ideal, compensates the photon phase fluctuations, without affecting the photon intensity, so that the phase state, at all times, fulfills the steadystate oscillation condition. This results in a sign change of the external cavity part of $f_{3}, f_{4}$ as compared to $f_{2}, f_{1}$. A significant reduction of the phase noise is expected, whereas

' The combined cavity incorporates both the internal and external cavity of the laser system but not the influence of the gain medium 


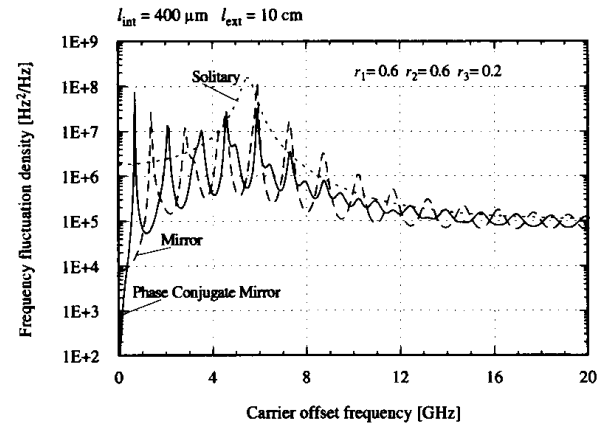

Fig. 3. Frequency fluctuation spectral density versus carrier offset for a solitary DFB laser, a DFB laser with conventional feedback from a long cavity and a DFB laser with phase conjugate feedback from a long cavity.

only the intensity noise originating from phase to intensityconverted noise will be reduced. The latter assumption agrees well with the results obtained in [16] and [19] on intensity noise in phase conjugated laser systems.

In the remaining part of this paper we discuss the significance of the aforementioned results on the laser phase noise behavior by investigating the frequency fluctuation spectrum as well as the phase fluctuation spectrum for various configurations of the laser system in Fig. 2.

\section{INFLUENCE OF PHASE CONJUGATE FEEDBACK ON LASER PHASE NOISE}

Comparing the characteristic functions of the external cavity for the two different configurations, we expect a significant difference in their phase noise behavior. To investigate this aspect, we calculate both the frequency fluctuation spectrum $S_{f}\left(f_{\mathrm{b}}\right)$ and the phase fluctuation spectrum $S_{\phi}\left(f_{\mathrm{b}}\right)$ for different configurations of the system in Fig. 2.

Here, these spectra are depicted as single-sideband spectra in the frequency domain as found from the double sideband frequency fluctuation spectrum in the angular frequency domain (14)

$$
\begin{aligned}
S_{f}\left(f_{\mathrm{b}}\right) & =2 \pi^{2} \cdot S_{\dot{\phi}}\left(\omega_{\mathrm{b}}\right) \\
S_{\phi}\left(f_{\mathrm{b}}\right) & =\frac{S_{f}\left(f_{\mathrm{b}}\right)}{f_{\mathrm{b}}^{2}} .
\end{aligned}
$$

A bulk $1.55 \mu \mathrm{m}$ InGaAsP DFB semiconductor laser of length $l_{\text {int }}=400 \mu \mathrm{m}$ with cleaved facets, i.e., $r_{1}=r_{2}=$ 0.6 is used for the investigations. All material parameters of this laser are given in Appendix B. In the following, this laser is investigated with and without external feedback for a bias current of twice the threshold unless otherwise stated. Throughout this paper, threshold is that of the entire laser system.

The frequency fluctuation spectral density of the solitary laser is shown in Fig. 3. It is seen that the laser has a linewidth, as found at zero frequency offset, of $\Delta \nu=\pi \cdot S_{f}(0)=$ $\pi \cdot 1.9 \cdot 10^{6}=6 \mathrm{MHz}$. The linewidth enhancement factor $\alpha$ given by the relation $1+\alpha^{2}=S_{f}(0) / S_{f}(\infty)$, is found to be 4.4. Finally, the relaxation oscillation frequency, $f_{R}$, of $5.5 \mathrm{GHz}$ is read at the peak in the frequency fluctuation

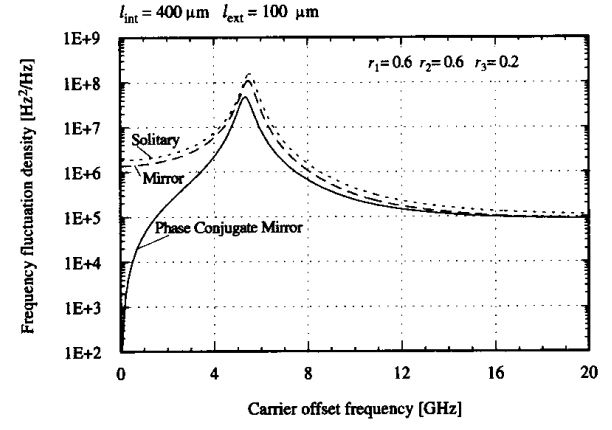

Fig. 4. Frequency fluctuation spectral density versus carrier offset for a solitary DFB laser, a DFB laser with conventional feedback from a short cavity, and a DFB laser with phase conjugate feedback from a short cavity.

spectrum. In the remaining part of this paper, it is investigated how the frequency noise of this laser behaves when either conventional external feedback or external PCF are applied.

Long cavity configurations of several centimeters length are typically used in conventional external cavity semiconductor lasers. Frequency fluctuation spectra of such a $10 \mathrm{~cm}$ external cavity laser with either conventional feedback or PCF of equal feedback fraction, $r_{3}=0.2$ are also shown in Fig. 3. It is seen that in the case of conventional feedback, a quite typical behavior is obtained. The linewidth of the laser is reduced to $25 \mathrm{kHz}$, and resonance peaks appear with a spacing determined by a multiple of the external cavity roundtrip time. It turns out, that the laser with PCF behaves quite differently. At low frequencies, the frequency noise drops towards zero due to the phase compensating nature of the PCM. A downward shift of the position of the resonance peaks by a factor of two is observed at low frequency-offsets. Further, an appearance of additional resonance peaks, that, in frequency, corresponds to a multiple of twice the external cavity roundtrip time, is observed at high frequency-offsets. This atypical behavior is attributed to the fact that a laser cavity with a PCM supports double roundtrip modes [7].

The special low-frequency behavior of the long-cavity phase conjugate laser leads us to investigate the same configuration for extremely short external cavities $\left(l_{\text {ext }}=100 \mu \mathrm{m}\right)$ corresponding to a butt-coupled PCM. The frequency noise behavior of this type of lasers are shown in Fig. 4 for the cases of conventional feedback and PCF, respectively. It is clearly seen that the PCF drastically reduces the frequency noise below the relaxation oscillation frequency. This phenomenon occurs because the PCM reverts the phase and thereby tracks the phase fluctuations within the PCM response bandwidth.

Since the PCM here is assumed ideal with instantaneous response, it might be expected that the PCM should reduce all the frequency noise. However, as seen, this is not the case. The PCM compensates the phase state of the photons to correct for the frequency noise which is induced by the spontaneous carrier recombinations. This can, naturally, not take place at any rate faster than the maximum interplay rate between carriers and photons. Consequently, frequency noise reduction only takes place at frequencies below the 


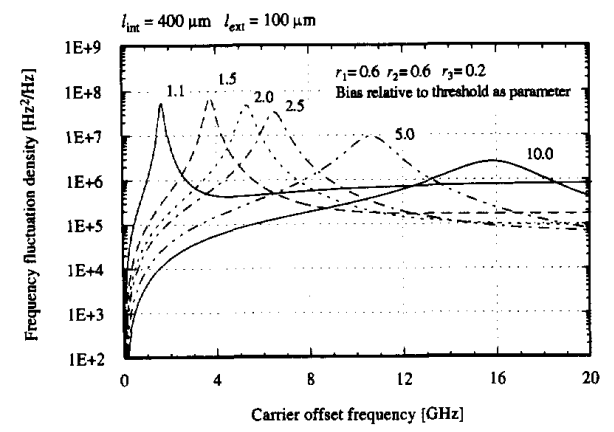

Fig. 5. Frequency fluctuation spectral density versus carrier offset for a DFB laser with phase conjugate feedback from a short cavity. The bias relative to threshold is used as parameter in order to vary the frequency of the relaxation oscillation.

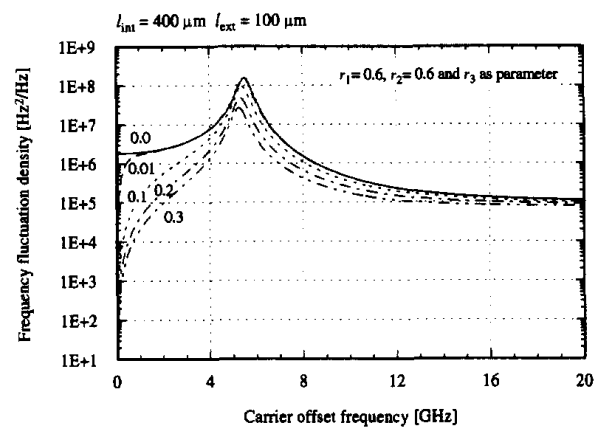

Fig. 6. Frequency fluctuation spectral density versus carrier offset for a DFB laser with phase conjugate feedback from a short cavity with the phase conjugate feedback fraction as parameter.

relaxation oscillation frequency. This gives rise to two clear facts: The PCM response does not need to be any faster than the relaxation oscillation, and to obtain the lowest noise, the relaxation frequency should be as high as possible. Varying the relaxation frequency (by varying the bias), it is possible to verify the aforementioned considerations. The results in Fig. 5 clearly show that the noise reduction bandwidth strongly depends on the relaxation frequency and relaxation damping, and thereby the bias of the laser. The higher the relaxation oscillation frequency, the lower the overall frequency noise.

The influence of the PCM response time is not investigated here since it simply limits the reduction bandwidth. Recently, both theoretical [22], [23] and experimental [22], [24] investigations have indicated possible PCM response times in the order of nanoseconds using MQW semiconductor media.

Another factor which limits the noise reduction bandwidth is the external feedback fraction $r_{3}$, as illustrated in Fig. 6. The higher the feedback, the lower the overall frequency noise. Feedback fractions of up to $11 \%$ have been achieved for self-pumped PCMs in semiconductor media [25]-[28] and even higher for externally pumped PCMs in semiconductor media [29].

It turns out, that the optimum frequency noise reduction is achieved if the laser is constructed with an AR-coated end facet $\left(r_{2}=0\right)$ and a butt-coupled PCM. This is illustrated

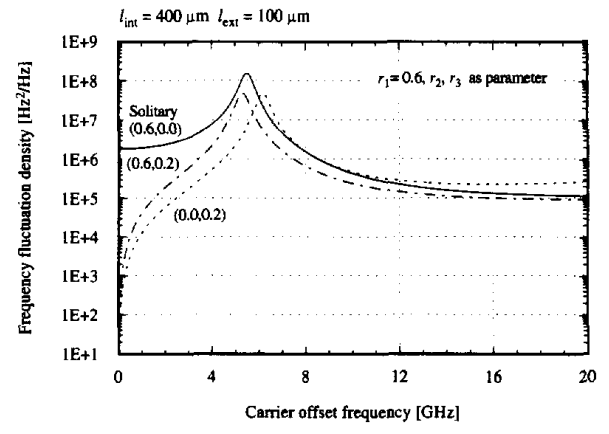

Fig. 7. Frequency fluctuation spectral density versus carrier offset for a solitary DFB laser $\left(r_{2}=0.6, r_{3}=0.0\right)$, a DFB laser with cleaved facets and phase conjugate feedback from a short cavity $\left(r_{2}=0.6, r_{3}=0.2\right)$, and a DFB laser with an ideally coated right facet and phase conjugate feedback from a short cavity $\left(r_{2}=0.0, r_{3}=0.2\right)$.

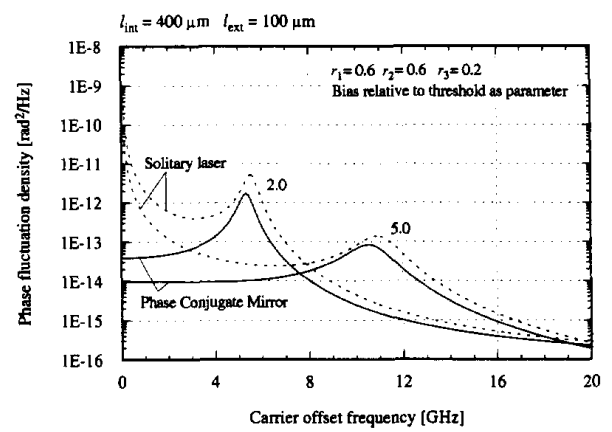

Fig. 8. Phase fluctuation spectral density versus carrier offset for a solitary DFB laser and for a DFB laser with phase conjugate feedback from a short cavity.

in Fig. 7 where the frequency fluctuation spectral density is given for the solitary laser, for the laser (as cleaved) with external PCF and for the laser with AR-coated end facet and external PCF. Since the external cavity in length is matched to compensate for the difference in refractive index, the last case approximates the situation of a solitary laser with one normal facet and one PCM facet. In this optimum configuration, multiple reflections are not present, and the model can be used to investigate strong feedback fractions as well. Stronger feedback fractions of more than the $30 \%$ investigated here will simply improve the noise reduction. Such strong PCF fractions are, however, very difficult to obtain.

To investigate the nature of the phase-locking performed by the PCF, the phase fluctuation spectral density is shown in Fig. 8 for two different bias conditions. At low frequencies the freequency noise drops very rapidly towards zero, cf. Fig. 7. The rate at which the noise drops is important in determining the nature of the noise reduction. For a solitary laser, the phase noise increases with decreasing carrier offset frequency as $f^{-2}$, cf. Fig. 8. However, when the PCF is present, the phase noise takes a finite value at zero carrier offset as normally achieved by first-order phase locking. This shows that by using PCF, it is possible to obtain a firstorder self-phase-locking of the laser. The zero carrier offset 


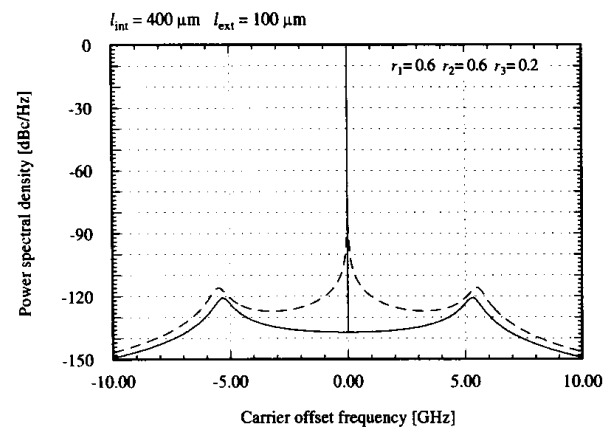

Fig. 9. Power spectral density of the emitted laser signal for a solitary DFB laser (dashed curve) and for a DFB laser with phase conjugate feedback from a short cavity (solid curve).

phase noise level depends on the initial linewidth of the solitary laser as well as the actual noise reduction bandwidth. Considering today's lasers with $1-10 \mathrm{MHz}$ linewidths or less, the reported nanosecond response times are far adequate to obtain an excellent noise performance. The dependency on the noise reduction bandwidth is illustrated by varying the bias and thereby the relaxation frequency. The phase variance, which is the integral of the phase fluctuation spectrum, is a measure of the total phase noise and is as low as $0.0017 \mathrm{rad}^{2}$ and $0.0003 \mathrm{rad}^{2}$ in the entire offset band of $20 \mathrm{GHz}$ for the bias current of two and five times the threshold, respectively.

In the power spectral domain, the consequence of this self-phase-locking is a drastic line narrowing. The resulting spectrum for a bias of twice the threshold, as shown in Fig. 9, consists of a very clean carrier peak with a noise level as low as $-137 \mathrm{dBc} / \mathrm{Hz}$ well below the relaxation oscillation frequency. For a bias of five times the threshold, the noise level is as low as $-143 \mathrm{dBc} / \mathrm{Hz}$. The spectral width and shape of the carrier peak depends on different external factors. Ideally, the peak is a delta function as we have only considered white frequency noise at low frequency-offsets. Higher order noise such as $1 / f$ frequency noise will, however, broaden the peak slightly. Further, if the PCM is externally pumped, the frequency noise of the pump sources will transfer directly to the phase conjugate laser and thereby set a lower limit to the spectral width. This indicates that, the PCM perferably should be self-pumped.

Finally, it should be noted, that the same typical behavior of the noise spectra is also achieved for Fabry-Perot lasers. This has been investigated by setting the coupling coefficient of the DFB laser model to zero.

\section{CONCLUSION}

A theoretical expression for the frequency/phase noise spectrum of semiconductor lasers with PCF has been derived. The theoretical derivation is based on a single-mode transmissionline description of semiconductor lasers with relatively weak external optical feedback. Further, the PCM giving the PCF is assumed ideal with instantaneous response. This assumption is considered reasonable since nanosecond PCM response times have been observed in MQW semiconductor materials.
The obtained expression for the frequency noise of lasers with PCF as compared to conventional feedback differ only in four simple functions describing the influence of the combined laser cavity. From these, it is seen that the PCF at steady-state locks the phase of the light to a constant value governed by a phase shift occurring at the PCM.

Calculations of the frequency noise spectrum have been performed for laser systems with both long and short external cavities. These show that, even for butt-coupled PCMs, the frequency noise below the relaxation oscillation of the laser is drastically reduced. Close to the carrier, the frequency noise actually decreases toward zero. It is found that the reduction bandwidth, assuming an ideal PCM, is governed by the relaxation oscillation frequency of the laser and by the amount of external feedback.

By investigating the phase noise spectrum instead of the frequency noise spectrum, we find that the action of the PCF corresponds to a state of first-order self-phase-locking. The resulting phase variance of the signal from the laser with PCF, in a $20 \mathrm{GHz}$ noise bandwidth, is well below $0.002 \mathrm{rad}^{2}$. This should be compared to the solitary laser where the phase variance is infinite.

The action of the PCF is also clearly seen in the power spectrum. The spectral shape of the laser does not remain Lorentzian but collapses to a narrow peak with a close to carrier noise level of less than $-137 \mathrm{dBc} / \mathrm{Hz}$. Ideally, the peak is a delta function, however, the spectral width of the peak will, in practice, be limited by higher order noise such as $1 / f$ frequency noise. Further, if an externally pumped PCM is used, the spectral width of the pump sources would limit the spectral width of the peak.

The theoretical results obtained in this paper are of great importance for applications where highly stable laser signals are required and where semiconductor lasers are preferred due to compactness. The PCM offers an elegant method for all optical phase noise reduction. With PCMs in MQW semiconductor materials, it might be possible in the future to implement monolithic phase conjugated semiconductor lasers yielding signals with extremely low phase-noise.

\section{APPENDIX A}

\section{FREQUENCY FLUCTUATION SPECTRUM OF SEMICONDUCTOR LASERS WITH EXTERNAL PHASE CONJUGATE FEEDBACK}

In this appendix, the expression for the frequency fluctuation spectrum is derived for semiconductor lasers with PCF. Such an expression does, to the best of the authors' knowledge, not exist in the literature. Here, we show that such an expression can also be derived from the linearized set of rate equations. It turns out that the expression is quite similar to that of semiconductor lasers with feedback from a conventional mirror, as given in Section III. The only difference appears in four characteristic functions describing the laser system cavity as explained in Section III.

The rate equation description of the semiconductor laser with PCF differs only from that of the conventional feedback in the description of the boundary condition related to the right facet. As described in Section II, this boundary condition is 
given as (9)

$$
A^{-}(t)=r_{2} A^{+}(t)+r_{3}\left(1-r_{2}^{2}\right) A^{+*}(t-\tau)
$$

where the factor $\phi_{\mathrm{PCM}}$ has been set to zero.

Linearizing $A^{-}(t)$ and $A^{+}(t)$ around the stationary solutions and transforming to the $s$-domain, the boundary condition becomes

$$
\delta A^{-}(s)=r_{2} \delta A^{+}(s)+r_{3}\left(1-r_{2}^{2}\right) \delta A^{+*}(-s) e^{-s \tau} .
$$

The linearized field equation in the $s$-domain is found from [20]:

$$
\begin{aligned}
F(s)= & s \delta A^{+}(s)-\frac{1}{2} C G_{N} \delta N(s) A_{\mathrm{s}}^{+} \\
& -f_{\mathrm{D}}\left[r_{\mathrm{L}} \delta A^{-}(s)-\delta A^{+}(s)\right] .
\end{aligned}
$$

Inserting (26), (27) becomes

$$
\begin{aligned}
F(s)= & \delta A^{+}(s)\left[s-f_{\mathrm{D}} r_{\mathrm{L}} r_{2}+f_{\mathrm{D}}\right] \\
& -\frac{1}{2} C G_{N} \delta N(s) A_{s}^{+} \\
& -f_{\mathrm{D}} r_{\mathrm{L}} r_{3}\left(1-r_{2}^{2}\right) \delta A^{+*}(-s) e^{-s \tau} .
\end{aligned}
$$

Equation (11) is still valid for external PCF, since the derivation of the expression is only based on the connection between the amount of photons in the laser cavity and the electric field at the first mirror [20]. The linearization of (11) gives the following two relations [20]:

$$
\begin{aligned}
P_{\mathrm{s}} & =\sigma\left|A_{\mathrm{s}}^{+}\right|^{2} \\
\delta P(t) & =\sigma 2 A_{\mathrm{s}}^{+} \delta\left|A^{+}(t)\right| .
\end{aligned}
$$

Describing $A^{+}(t)$ as

$$
A^{+}(t)=\left|A^{+}(t)\right| e^{j \phi(t)}
$$

linearizing it to the first-order, inserting (29) and (30), and transforming to the $s$-domain gives

$$
\delta A^{+}(s)=\frac{A_{\mathrm{s}}^{+}}{2 P_{\mathrm{s}}}\left\{\delta P(s)+2 j P_{\mathrm{s}} \delta \phi(s)\right\}
$$

By complex conjugating (32) and changing the sign of $s$, we find

$$
\delta A^{+*}(-s)=\frac{A_{\mathrm{s}}^{+}}{2 P_{\mathrm{s}}}\left\{\delta P(s)-2 j P_{\mathrm{s}} \delta \phi(s)\right\} .
$$

Using these relations for $\delta A^{+}(s)$ and $\delta A^{+*}(-s)$ in the field equation (28) gives

$$
\begin{aligned}
F(s)= & \frac{A_{\mathrm{s}}^{+}}{2 P_{\mathrm{s}}}\left\{\delta P(s)+j 2 P_{\mathrm{s}} \delta \phi(s)\right\}\left[s-f_{\mathrm{D}} r_{\mathrm{L}} r_{2}+f_{\mathrm{D}}\right] \\
& -\frac{1}{2} C G_{N} \delta N(s) A_{\mathrm{s}}^{+} \\
& -f_{\mathrm{D}} r_{\mathrm{L}} r_{3}\left(1-r_{2}^{2}\right) \frac{A_{\mathrm{s}}^{+}}{2 P_{\mathrm{s}}}\left\{\delta P(s)-j 2 P_{\mathrm{s}} \delta \phi(s)\right\} e^{-\tau s}
\end{aligned}
$$

The Langevin noise function $F(s)$ can be divided into an amplitude $F_{P}(s)$ and phase $F_{\phi}(s)$ part using the correlation relations for the Langevin noise functions

$$
\begin{aligned}
& F_{P}(s)=\frac{P_{\mathrm{s}}}{A_{\mathrm{s}}^{+}}\left\{F(s)+F^{*}(-s)\right\} \\
& F_{\phi}(s)=-\frac{j P_{\mathrm{s}}}{2 A_{\mathrm{s}}^{+}}\left\{F(s)-F^{*}(-s)\right\} .
\end{aligned}
$$

TABLE I

Laser Parameters

\begin{tabular}{|c|c|l|}
\hline Symboi & Value & Name \\
\hline \hline$a_{1}$ & $2 \cdot 10^{8} \mathrm{~s}^{-1}$ & Recombination constant \\
\hline$b_{1}$ & $1.5 \cdot 10^{-16} \mathrm{~m}^{3} / \mathrm{s}$ & Recombination constant \\
\hline$c_{1}$ & $1.54 \cdot 10^{-40} \mathrm{~m}^{6} / \mathrm{s}$ & Recombination constant \\
\hline$\alpha_{\mathrm{i}}$ & $2.7 \cdot 10^{3} \mathrm{~m}^{-1}$ & Internal loss pr. unit length \\
\hline$\beta_{0}$ & $13.872 \cdot 10^{5} \mathrm{~m}^{-1}$ & $\pi / \Lambda$, where $\Lambda$ is the grating period \\
\hline$\phi_{0}$ & 0 & Phase of the grating at $z=0$ \\
\hline$N_{0}$ & $0.9 \cdot 10^{24} \mathrm{~m}^{-3}$ & Carrier density at transparency \\
\hline$\gamma_{N}$ & $2.9 \cdot 10^{-20} \mathrm{~m}^{2}$ & Gain coefficient \\
\hline$\gamma_{\omega}$ & $6.9 \cdot 10^{-24} \mathrm{~s}^{2} \mathrm{~m}^{1}$ & Gain coefficient \\
\hline$\frac{d_{w}}{d N}$ & $2.12 \cdot 10^{-11} \mathrm{~s}^{-1} \mathrm{~m}^{3}$ & Angular frequency change with carrier density \\
\hline$\omega_{\mathrm{p}}$ & $1.2161 \cdot 10^{15} \mathrm{rad} / \mathrm{s}$ & Reference frequency at transparency \\
\hline$n_{0}$ & 3.3 & Reference refractive index \\
\hline$n_{p p}$ & 2.0 & Spontaneous emission factor \\
\hline$\frac{d n}{d N}$ & $-1 \cdot 10^{-26} \mathrm{~m}^{3}$ & Refractive index dependency of $N$ \\
\hline$\kappa_{g}$ & $5 \cdot 10^{3} \mathrm{~m} \mathrm{~m}^{-1}$ & Coupling coefficient \\
\hline$n_{g}$ & 3.75 & Group index \\
\hline$d$ & $0.15 \mu \mathrm{m}$ & Thickness of active layer \\
\hline$w$ & $3.3 \mu \mathrm{m}$ & Width of the active layer \\
\hline 1 & $400 \mu \mathrm{m}$ & Length of the active layer \\
\hline & & \\
\hline
\end{tabular}

From this, we find the following set of linearized rate equations:

$$
\left[\begin{array}{ccc}
s+f_{1} & 2 P_{\mathrm{s}} f_{2} & -C_{\mathrm{r}} G_{N} P_{\mathrm{s}} \\
\frac{-f_{3}}{2 P_{\mathrm{s}}} & s+f_{4} & -\frac{1}{2} C_{\mathrm{i}} G_{N} \\
\frac{\omega_{\mathrm{R}}^{2}}{G_{N} P_{\mathrm{s}}} & 0 & s+\frac{1}{\tau_{\mathrm{R}}}
\end{array}\right]\left[\begin{array}{c}
\delta P(s) \\
\delta \phi(s) \\
\delta N(s)
\end{array}\right]=\left[\begin{array}{c}
F_{P}(s) \\
F_{\phi}(s) \\
F_{N}(s)
\end{array}\right]
$$

where the characteristic functions of the external cavity are given as

$$
\begin{aligned}
& \begin{aligned}
& f_{1, \mathrm{PCM}}= \operatorname{Re}\left(g_{1}\right)-0.5 r_{3}\left(1-r_{2}^{2}\right)\left(g_{2}+g_{2}^{*}\right) e^{-j \omega_{\mathrm{b}} \tau} \\
& f_{2, \mathrm{PCM}}=-\operatorname{Im}\left(g_{1}\right)+0.5 j r_{3}\left(1-r_{2}^{2}\right)\left(g_{2}-g_{2}^{*}\right) e^{-j \omega_{\mathrm{b}} \tau}(39) \\
& f_{3, \mathrm{PCM}}=-\operatorname{Im}\left(g_{1}\right)-0.5 j r_{3}\left(1-r_{2}^{2}\right)\left(g_{2}-g_{2}^{*}\right) e^{-j \omega_{\mathrm{b}} \tau} \\
& f_{4, \mathrm{PCM}}= \operatorname{Re}\left(g_{1}\right)+0.5 r_{3}\left(1-r_{2}^{2}\right)\left(g_{2}+g_{2}^{*}\right) e^{-j \omega_{\mathrm{b}} \tau} . \\
& \text { The expression for the double sideband frequency fluctuation } \\
& \text { spectrum is derived following the procedure of }[21]: \\
& s_{\dot{\phi}}\left(\omega_{\mathrm{b}}\right)=\frac{R_{\mathrm{sp}} \omega_{\mathrm{b}}^{2}}{2 P_{\mathrm{s}}|D(s)|^{2}}\left\{\left|\left(s+f_{1}\right)\left(s+\frac{1}{\tau_{\mathrm{R}}}\right)+C_{\mathrm{r}} \omega_{\mathrm{R}}^{2}\right|^{2}\right. \\
&\left.\quad+\left|\left(s+\frac{1}{\tau_{\mathrm{R}}}\right) f_{3}-C_{\mathrm{i}} \omega_{\mathrm{R}}^{2}\right|^{2}\right\} .
\end{aligned}
\end{aligned}
$$

It should be emphasized, that the set of linearized rate equations (37), and the expression for the frequency fluctuation spectrum (42) are general. It is only the characteristic functions that differ from system to system.

\section{APPENDIX B}

\section{Laser Material Parameters}

In this appendix, the material parameters of the laser which has been used in the calculations are listed in Table $I$.

\section{ACKNOWLEDGMENT}

B. Tromborg and J. Mørk from the Danish Telecommunication Research Laboratory, R. J. S. Pedersen at the Electromagnetics Institute at the Technical University of Denmark, P. M. Petersen at the Physics Institute at the Technical University of Denmark, and L. Eskildsen at AT\&T Bell Laboratories are all gratefully acknowledged for fruitful and inspiring discussions. 


\section{REFERENCES}

[1] J. M. Kahn, C. A. Burrus, and G. Raybon, "High-stability $1.5 \mu \mathrm{m}$ external-cavity semiconductor lasers for phase-lock applications," IEEE Photon. Technol. Lett., vol. 1, no. 7, pp. 159-161, 1989.

[2] F. Bertinetto, P. Gambini, R. Lano, and M. Puleo, "Stabilization of the emission frequency of $1.54 \mu \mathrm{m}$ DFB laser doides to hydrogen iodide," IEEE Photon. Technol. Lett., vol. 4, no. 4, pp. 472-474, 1993.

[3] M. Ohtsu, M. Murata, and M. Kourogi, "FM noise reduction and subkilohertz linewidth of an AIGaAs laser by negative electrical feedback," IEEE J. Quantum Electron., vol. QE-26, no. 2, pp. 231-241, 1990.

[4] M. Tamburrini, M. Parent, L. Goldberg, and D. Stillwell, "Optical feed for a phased array microwave antenna," IEE Electron. Lett., vol. 23, no. 13 , pp. $680-681,1987$.

[5] L. Goldberg, A. M. Yurek, H. F. Taylor, and J. F. Weller, " $35 \mathrm{GHz}$ microwae signal generation with an injection-locked laser diode," IEE Electron. Lett., vol. 21, no. 18, pp. 814-815, 1985.

[6] U. Gliese, T. N. Nielsen, M. Bruun, E. L. Christensen, and K. E. Stubkjær, S. Lindgren, and B. Broberg, "A wideband heterodyne optical phase locked loop for generation of $3-18 \mathrm{GHz}$ microwave carriers," IEEE Photon. Technol. Lett., vol. 4, no. 8, pp. 936-938, 1992.

[7] J. Auyeung, D. Fekete, D. M. Pepper, and A. Yariv, "A theoretical and experimental investigation of the modes of optical resonators with phase-conjugate mirrors," IEEE J. Quantum Electron., vol. QE-15, no. 10 , pp. $1180-1188,1979$.

[8] K. Vahala, K. Kyuma, A. Yariv, S.-K. Kwong, M. Cronin-Golomb, and K. Y. Lau, "Narrow linewidth, single-frequency semiconductor laser with a phase conjugate external cavity mirror," Appl. Physics Lett., vol. 49 , no. 23 , pp. $1563-1565,1986$.

[9] R. R. Stephens, R. C. Lind, and C. R. Giuliano, "Phase conjugate master oscillator-power amplifier using $\mathrm{BaTiO}_{3}$ and $\mathrm{AlGaAs}$ semiconductor diode lasers," Appl. Physics Lett., vol. 50, no. 11, pp. 647-647, 1987.

[10] S. Weiss, M. Segev, and B. Fischer, "Line narrowing and self-frequency scanning of laser diode arrays coupled to a photorefractive oscillator," IEEE J. Quantum Electron., vol. QE-24, no. 5, pp. 706-708, 1988.

[11] M. Segev, Y. Ophir, B. Fischer, and G. Eisenstein, "Mode locking and frequency tuning of a laser diode array in an extended cavity with a photorefractive phase conjugate mirror," Appl. Physics Lett., vol. 57, no. 24 , pp. $2523-2525,1990$.

[12] S. MacCormack, J. Feinberg, and M. H. Garrett, "Self-aligning injection locking of a laser diode array," in Proc. Technical Digest 13th Conf. Lasers and Electrooptics, Baltimore, MD, pp. 532-533.

[13] Y. Champagne, N. McCarthy, and R. Tremblay, "Optical phaseconjugate feedback effects on gain-guided diode laser characteristics," IEEE J. Quantum Electron., vol. QE-25, no. 3, pp. 595-601, 1989.

[14] G. P. Agrawal and J. T. Klaus, "Effect of phase-conjugate feedback on semiconductor laser dynamics," Optics Lett., vol. 16, no. 17, pp. 1325-1327, 1991

[15] G. H. M. van Tartwijk, H. J. C. van der Linden, and D. Lenstra, "Theory of a diode laser with phase-conjugate feedback," Optics Lett., vol. 17, no. 22 , pp. $1590-1592,1992$

[16] G. P. Agrawal and G. R. Gray, "Effect of phase-conjugate feedback on the noise characteristics of semiconductor lasers," Phys. Rev. A, vol. 46, no. 9 , pp. $5890-5898,1992$.

[17] G. P. Agrawal, D. Huang, and G. R. Ray, "Effect of phase-conjugate feedback on the intensity and phase noise of semiconductor lasers," in Proc. Techn. Digest 13th Conf. Lasers Electrooptics, Baltimore, MD, 1993, pp. 318-319.

[18] L. N. Langley and K. A. Shore, "Intensity noise in laser diodes with phase-conjugate optical feedback," in Proc. Digest 13th Conf. Lasers Electrooptics, Baltimore, MD, 1993, pp. 516-517.

[19] L. N. Langley and K. A. Shore, "Effect of phase-conjugate optical feedback on the intensity noise in laser diodes," Optics Lett., vol. 18, no. 17 , pp. $1432-1434,1993$.

[20] B. Tromborg, H. Olesen, X. Pan, and S. Satio, "Transmission line description of optical feedback and injection locking for Fabry-Perot and DFB lasers," IEEE J. Quantum Electron., vol. QE-23, no. 11, pp. 1875-1889, 1987.

[21] P. Spano, S. Piazzolla, and M. Tamburrini, "Theory of noise in semi conductor lasers in the presence of optical feedback," IEEE J. Quantum Electron., vol. QE-20, no. 4, pp. 350-357, 1984.
[22] R. G. Lindquist, P. G. LoPresti, and I. C. Khoo, "Theory and experiments on picosecond multiwave-mixing in GaAs," in Proc. Digest 13th Conf. Lasers Electrooptics, Baltimore, MD, 1993, pp. 342-344.

[23] V. V. Shkunov and M. V. Zolotarev, "Theory of the photo-refractive effect for MQM structures in reflecting geometry," In: Proc. Digest 13th Conf. Lasers Electrooptics, Baltimore, MD, 1993, pp. 528-530.

[24] Y. Zhao, C. Wu, P. Shah, M. K. Kim, and L. R. Dawson, "Experimental observation of optical phase conjugation in InGaAs/GaAs multiple quantum wells at $1.06 \mu \mathrm{m}$ wavelength," in Proc. Digest 13th Conf. Lasers Electrooptics, Baltimore, MD, 1993, p. 516.

[25] R. B. Bylsma, A. M. Glass, D. H. Olson, and M. Cronin-Golomb, "Selfpumped phase conjugation in InP:Fe," Appl. Physics Lett., vol. 54, no. 20, pp. 1968-1970, 1989.

[26] P. L. Chua, D. T. H. Liu, and L. J. Cheng, "Self-pumped and double phase conjugation in GaAs with applied dc electric field," Appl. Physics Lett., vol. 57, no. 9, pp. 858-860, 1990.

[27] J. E. Millerd and E. M. Garmire, "Self-pumped phase conjugation in InP:Fe using band-edge resonance and temperature stabilization: Theory and experiments," Optics Lett., vol. 17, no. 2, pp. 100-102, 1992.

[28] M. L. Minden, D. M. Pepper, and M. B. Klein, "Self-pumped phase conjugator/retromodulator using InP:Fe at $1.3 \mu \mathrm{m}$," in Proc. Techn. Digest 12th Conf. Lasers Electrooptics, Anaheim, CA, 1992, pp. 58-60.

[29] V. Vieux, P. Gravey, N. Wolffer, and G. Picoli, "Double-phase conjugated mirror and double color pumped oscillator in photorefractive InP:Fe," Appl. Physics Lett., vol. 58, no. 25, pp. 2880-2882, 1991.

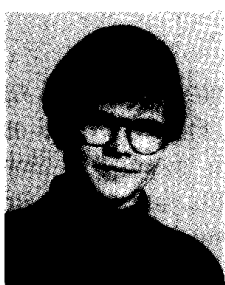

Lykke Petersen was born in 1967. She received the M.Sc. degree in electrical engineering from the Electromagnetics Institute, Technical University of Denmark in 1993, on modeling of semiconductor lasers with phase conjugate feedback. Her research activities have covered modeling and characterization of semiconductor lasers.

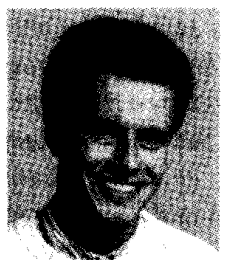

Ulrik Gliese was born in 1965 . He received the M.Sc. and Ph.D. degrees from the Electromagnetics Institute, Technical University of Denmark, in 1989 and 1992, respectively. From 1989 to 1992 he has been working as a Research Associate at the Electromagnetics Institute and since 1992 as a Senior Researcher. His research activities have covered coherent optical communication systems, microwave devices, characterization of advanced semiconductor lasers, methods for laser phase noise reduction, optical phase locked loops and phase conjugate feedback, optical modulation, optoelectronic MMIC devices, and optical microwave links.

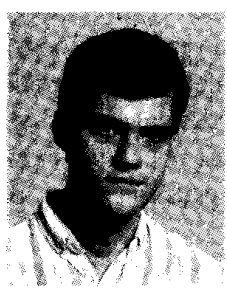

Torben Nørskov Nielsen was born in $1965 . \mathrm{He}$ received the M.Sc. and Ph.D. degrees from the Electromagnetics Institute, Technical University of Denmark, in 1991 and 1994, respectively. Since 1991, he has been working as a Research Associate at the Electromagnetics Institute. His research activities have covered microwave amplifiers, modeling and characterization of semiconductor optical amplifiers, optical modulation with semiconductor optical amplifiers, optical phase-locked loops and optical microwave links. 